\title{
Novas Espécies da Flora Amazônica
}

\author{
WIILIAM A. RODRIgUeS (*) \\ Marlene Freitas dA Silva (*) \\ Instituto Nacional de Pesquisas \\ da Amazônia
}

Por omissão alheia a nossa vontade as no. vas espécies Vochysia rufescens W. Rodr. $\epsilon$ Rauia prancei W. Rodr. \& M. F. da Silva publicadas no primeiro número da revista ACTA AMAZONICA sairam sem as diagnoses latinas, razão pela qual resolvemos apresentá-las novamente neste artigo.

\section{VOCHYSIACEAE}

Vochysia rufescens W. Rodrigues, n. sp.

= Vochysia rufescens W. Rodr., in Acta Amazonica 1(1):7-10 (1971), fig. 1. Nomen nudum.

Ad sectionem Pachyantha Stafl.. Arbor circa $25 \mathrm{~m}$ alta $35 \mathrm{~cm}$ diametro; truncus cylindricus, erectus sine radicibus tabularibus. Ramuli obsolete tetrangulosi juniores cum petiolis, foliorum pagina inferiore, inflorescentiis, bracteis et alabastris rufo-tomentosis; ramuli vetustiores teretes, ca. $0,5 \mathrm{~cm}$ diametro cortice cinereo. Stipulae triangulares subulatae, persistentes, $0,3-0,5 \mathrm{~cm}$ longae, $0,1-0,2$ $\mathrm{cm}$ latae, rufo-tomentosae deinde glabrescentes. Folia opposita petiolo vulgo $1,0-2,0 \mathrm{~cm}$ longo, 0,3-0,4 cm diametro, supra leviter sulcato; lamina vulgo $10-21 \mathrm{~cm}$ longa, $4-9,5 \mathrm{~cm}$ lata plus minusve obovato-oblonga vel oblonga ad apicem abrupte acuminata, acumine $1,5-2,0 \mathrm{~cm}$ longo, acuto apice proprio mucronulato ad basin obtusa vel breviter cuneata, rigide coriacea saepe longitudinaliter modice arcuata, concolor siccitate fuscescens, supra glabra nitidula; costa mediana, nervi secundarii, hervi marginales, venae venulaeque supra impressae infra prominentes; nervi secundarii 27-38 jugi retiusculo - adscentes, approximati ex angulo $60-70^{\circ}$; venulae reticulatae subtus prominulae, nervi marginales distincte inter nervorum secundariorum apices arcuati usque ad 1,5 $\mathrm{mm}$ proper marginem inserti. Inflorescentia terminalis cylindrica usque ad $36 \mathrm{~cm}$ longa, 0,3-0,4 cm diametro, densiflora rache apicem versus sensim attenuata ad basin ca. 0,5-0,7 cm diametro; cincinni 1-2 flori; bractae caducae, subulati usque ad $0,6 \mathrm{~cm}$ longae, $0,2 \mathrm{~cm}$ latae extus rufotomentosae intus glabrae, bracteolis similibus, 1-2 $\mathrm{mm}$ longis; pedunculi ca. $0,5-0,7 \mathrm{~cm}$ longi, $0,1 \mathrm{~cm}$ lati; pedicelli usque ad $1,2 \mathrm{~cm}$ longi, $0,1 \mathrm{~cm}$ crassi; alabastra recurva, obtusa, rufo-tomentosa, $1,2-1,8 \mathrm{~cm}$ longa, $0,5 \mathrm{~cm}$ lata calcare crasso ca. $1,0 \mathrm{~cm}$ longo, $0,2-0,3 \mathrm{~cm}$ diametro ad basin constricto, inflato, incurvo. Flores crassi in vivo flavi, siccitate extus rufotomentosi, intus glabri; calicis laciniae 4 eis anterioribus $0,3-0,5 \mathrm{~cm}$ longis, obtusis, intus glabris, lacinia postica praefloratione ca. 0,5-0,6 cm longa; anthera siccitate $3,5 \mathrm{~mm}$ longa, $2 \mathrm{~mm}$ lata (in vivo $6-7 \mathrm{~mm} \times 3 \mathrm{~mm}$ ) crassa dorso piloso, apice glabro, obtuso cucullato, filamento ca. $2 \mathrm{~mm}$ longo; flores communiter staminodiis deficientes; staminodium ( 1 tantum visum) $0,5 \mathrm{~cm}$ longum, 0,1 $\mathrm{cm}$ latum, ligulatum, pilosum, ad marginem ciliatum; pistillum pilosum stilo supra medium glabro, cylindrico, crasso, siccitate ca. $0,3-0,5 \mathrm{~cm}$ longo, $0,1 \mathrm{~cm}$ lato, stigmate capitato ca. $0,1 \mathrm{~cm}$ diametro; ovarium piramidale, rufo-tomentosum ca. $0,2 \mathrm{~cm}$ altum et latum. Capsula usque ad $6,0 \mathrm{~cm}$ longa, 2,5 $\mathrm{cm}$ lata, oblonga, glabra, verruculosa, in vivo atrovirescens, siccitate nigrescens, glabra, oblonga, longitudinaliter sulcata, 3-angulosa,

\footnotetext{
(*) - Bolsistas do Conselho Nacional de Pesquisas.
} 
angulis obtusis ad basin haud peculiariter incrassata. Semina rufescentia usque ad 5,5 $\mathrm{cm}$ longa, $1,5 \mathrm{~cm}$ lata alis rotundatis.

Habitat in Brasilia, Amazonas, via $\mathrm{Ma}$ naus-Itacoatiara, circa Reserva Florestal W. Egler, in silva non inundabili terrae argillosae, ubi non rara est. Typus W. A. Rodrigues 8671 (Holotypus INPA 27843, Isotypus MG) .

\section{$R U T A C E A E$}

Rauia prancei W. Rodr. \& M. F. Silva = Rauia prancei W. Rodr. \& M. F. Silva in Acta Amazonica 1(1) : 21-22 (1971), fig. 1 Nomen nudum.

Arbor parva $7 \mathrm{~m}$ alta, $10 \mathrm{~cm}$ diametro, rami glabri dense glandulosi. Folia membranacea, glabra, late lanceolata, supra nitida, subtus pallida atropunctulata punctis glandulosis, $12-14 \mathrm{~cm}$ (raro ad $18 \mathrm{~cm}$ ) longa, 5,5-7 (8) $\mathrm{cm}$ lata, apice acuto et acuminato, basi cuneata, margine integra et revoluta. Petiolus glabrus, 2,5-3 cm longus, $1,5 \mathrm{~mm}$ crassus; nervi mediani et secundarii utrinque impresse reticulati. Inflorescentia terminalis corymbosa, 13-15 cm longa; rachis longitudi- naliter striata, ramificatione dichotomica, ferruginea. Alabastra florifera longa cylindrica, praefloratione imbricata, $15 \mathrm{~mm}$ longa. Calyx campanulatus, 3-4 mm longus, saturate lepidotus, intus glabrus, rubescens, 5-lobatus; laciniae $0,5 \mathrm{~mm}$ longae, apice acuto. Fiores gamopetali extus ut intus puberuli ad 2,5 $\mathrm{cm}$ longi tubo 7,5-10 $\mathrm{mm}$ longo; petala 5 , alba liniaria, alternisepala margine vix crenulata. Stamina 7 (2 tantum fertilia), 7,5 mm longa, antheris oblongis $3-4 \mathrm{~mm}$ longis rimosis ad apicem obtusis; staminodia majora 3 , filiformis, quam petala $3.4 \mathrm{~mm}$ breviora. libera; staminodia 2 e basi $2 / 3$ longitudine petalis adnata. Ovarium globosum vel subglobosum 1-1,5 mm longum, profunde 5-lobulatum, 5loculare, disco membranoso $2 \mathrm{~mm}$ alto irregulariter laciniato circundatum. Stylus teres, filiformis, $2-3 \mathrm{~mm}$ longus pilis hispidis ubique vestitus; stigma glabrum, claviforme. Fructus ignoti.

Typus: Tree $7 \mathrm{~m} \times 10 \mathrm{~cm}$ diameter. Corola white. Forest on terra firme. Brasil, Territory of Rondonia, basin of rio Madeira, vicinity of São Lourenço mines, $65^{\circ} 6^{\prime}$ W; $9^{\circ} 33^{\prime}$ S. G.T. Prance, W. A. Rodrigues, J.F. Ramos \& L.G. Farias 8944 . Nov. 27/1968 (Holotypus INPA 25743, Isotypus MG 39624, NY.). 